\title{
Polysèmes
}

Revue d'études intertextuelles et intermédiales

\section{Le passage du poète Hart Crane}

\section{Béatrice Pire}

\section{(2) OpenEdition \\ Journals}

Édition électronique

URL : http://journals.openedition.org/polysemes/1633

DOI : 10.4000/polysemes. 1633

ISSN : 2496-4212

\section{Éditeur}

SAIT

Édition imprimée

Date de publication : 1 janvier 2003

Pagination : 187-197

ISSN : 0999-4203

\section{Référence électronique}

Béatrice Pire, «Le passage du poète Hart Crane », Polysèmes [En ligne], 6 | 2003, mis en ligne le 10 janvier 2017, consulté le 22 avril 2019. URL : http://journals.openedition.org/polysemes/1633 ; DOI : 10.4000/polysemes.1633

Ce document a été généré automatiquement le 22 avril 2019.

Polysèmes 


\title{
Le passage du poète Hart Crane
}

\author{
Béatrice Pire
}

1 John Unterecker a donné à sa biographie de Hart Crane le titre particulièrement approprié de «Voyager ", le voyageur ${ }^{1}$. De fait, à l'instar de son double français Arthur Rimbaud, auquel il a été maintes fois comparé, Crane fait figure de poète vagabond. Fuyant les tourmentes familiales de son Ohio natal, il gagne New York dès l'âge de dixsept ans, puis déambule entre les Caraïbes, la Californie et l'Europe. En 1929, il séjourne quelques mois à Paris, où il reste emprisonné pendant plusieurs jours pour ivresse sur la voie publique, puis dans le Sud de la France. En 1931, il obtient une bourse de la fondation Guggenheim et part vivre un an au Mexique. C'est en revenant à New York, en avril 1932, qu'il se jette dans le golfe du Mexique, un geste désespéré qui n'a cessé depuis soixantedix ans de nourrir le caractère légendaire de son existence. Voyageur infiniment déraciné, c'est également un homme instable, ou comme le soulignait Philippe Soupault, « un homme déchiré », brisé par le divorce de ses parents, alcoolique et suicidé. Composé en 1925 et inclus dans White Buildings, le premier recueil de Crane, un poème intitulé « Passage » est, dans une certaine mesure, à l'image de son auteur, le reflet textuel d'un perpétuel errant qui, lors d'une dernière traversée comme passager à bord d'un paquebot, trépasse en passant par-dessus bord ; la marque d'un homme marginal ou d'un artiste sans véritable attache et domicile fixe : un passenger, par excellence, dans le sens sociologique que lui accorde l'américain Victor Turner :

In a situation which is temporally liminal and spatially marginal the passengers in a protracted rite de passage are stripped of status and authority-in other words removed from a social structure [...]. Prophets and artists tend to be liminal and marginal people, edgemen who strive with a passionate sincerity to rid themselves of the clichés associated with status incumbery and roleplaying. ${ }^{2}$

Cherchant en permanence à échapper à toute structure sociale et à conserver une position liminale, Hart Crane inscrit dans ces vers sa propre marginalité, et façonne un texte où les mots s'arrachent aux structures syntaxiques et sémantiques imposées par la grammaire et la logique, un poème en conséquence glissant, et rebelle à la fixité de l'interprétation. Refusé à la publication par T.S. Eliot pour The Criterion et pour The Dial par Marianne Moore qui lui reprochait sa trop grande densité de contenu et son manque 
de simplicité, «Passage » est en effet difficile et nombreux sont les critiques qui s'y sont heurtés sans jamais pourtant réussir à en immobiliser le sens. Cette impuissance résulte du projet même de Crane qui était d'inventer un idiome fuyant (an elusive idiom) et un langage fluide $e^{3}$. Le titre du poème est donc programmatique puisqu'il annonce une parole passagère qu'on ne peut arrêter dans aucun signifié, des vers qui ne font que passer infiniment d'un signifié à l'autre.

Where the cedar leaf divides the sky

I heard the sea.

In sapphire arenas of the hills

I was promised an improved infancy.

Sulking, sanctioning the sun,

My memory I left in a ravine,

Casual louse that tissues the buckwheat,

Aprons rocks, congregates pears

In moonlit bushels

And wakens alleys with a hidden cough.

Le premier vers s'ouvre sur l'image d'une feuille de cèdre qui taille une brèche dans le ciel, interstice spatial qui par la synesthésie permet au poète d'entendre la voix de la mer et la promesse d'un retour à l'enfance. Les strophes laissent inaugurer un rite de passage, mais un rite inversé : il ne s'agit pas, comme dans la plupart de ces cérémonies, de passer de l'enfance à l'âge adulte, mais plutôt de régresser, ou comme l'indique l'étymologie (re : retour/gradi : marcher), de faire un pas en arrière vers la petite enfance (infancy), de retourner à l'état de silence antérieur au langage, à l'état de celui qui ne parle pas (in/ fans). Le passage promis est double : temporel (passage du temps à un hors-temps) et textuel (passage du langage au silence).

Cette double thématique est impulsée dans le premier vers par le syntagme de la feuille de cèdre : la feuille est une feuille métaphorique, moins végétale que textuelle, qui sera par la suite dépliée dans la forme plus littérale d'une biographie (a too well known biography), d'un livre (my stolen book; He closed the book) et finalement d'une page (the page). Portant cette feuille, le cèdre infléchit le vers dans un sens plus temporel, que prolonge à la strophe suivante le substantif memory. Comme souvent chez Crane, l'arbre doit être perçu dans sa figure symbolique d'arbre généalogique, comme lieu de mémoire ou recueil du passé. Dans un autre texte intitulé "Forgetfulness », une phrase déploie la même idée : «Forgetfulness is white,- white as a blasted tree ». Abattre l'arbre, c'est blanchir la mémoire et plonger dans l'oubli. La feuille du cèdre devient donc la métaphore de la page déjà écrite, de l'inscription antérieure qui ancre le je poétique dans une mémoire et une histoire auxquelles il tente d'échapper. Certains critiques ont insisté sur la valeur phonologique du préfixe ce dans le substantif cedar. La sonorité ferait écho dans le vers suivant au mot sea et dans le quatrième vers au suffixe cy du mot infancy. Cette connivence des trois termes fondée sur l'harmonie phonique s'annule cependant quand on recherche l'étymologie, certes contestée mais intéressante, de cedar qui viendrait du préfixe grec ked signifiant être noir de suie ou fumant. L'accord de cedar, sea et infancy serait donc un leurre sonore déjoué par l'étymologie qui sémantiquement associe davantage le conifère à l'adjectif composé chimney-sooted et au nom smoke dans la quatrième strophe, ainsi qu'aux métaphores de la mémoire développées dans les quatre derniers vers de la seconde strophe.

Encaissés entre le tiret et le point final, ces vers apposés à my memory, dessinent un espace typographiquement clos qui reflète l'enfermement du je poétique dans le ravin. Les 
verbes tissues et aprons attribuent à la mémoire une fonction de dissimulation visuelle qui contamine le sens même des vers, comme si le voile de blé noir avait aussi été jeté sur le texte devenu tissu d'obscurités grammaticales et sémantiques. On saisit mal le lien qui unit par exemple la mémoire, prise dans sa forme métaphorique de pou, et les poires assemblées dans les boisseaux. Le sens a priori obscur s'éclaircit cependant quand on sait que le terme bushel ne désigne pas seulement l'unité de mesure du fruit, mais aussi un récipient ou encore un tuyau permettant l'écoulement des eaux ou des fumées. Amorce de ravine, lit creusé par un ruissellement, le mot annonce aussi proleptiquement chimney, conduit de la quatrième strophe par lequel s'échappent les fumées. Le nom approfondit également le thème de la dissimulation déjà suggéré, puisqu'on peut reconstituer le puzzle du proverbe «to hide one's light under a bushel» qui signifie «cacher ses talents ", en en rassemblant les différentes pièces lit et hidden éparpillés dans les vers. La métaphore du boisseau dessinerait donc la mémoire sous les contours d'un vase fermé qui camouflerait la lumière et empêcherait les talents poétiques de s'exprimer.

Le nom pears est plus problématique que bushels. Peut- être désigne-t-il les fruits de la création condamnés à l'enfermement, hypothèse qui s'accorde avec l'interprétation précédente. Il est également possible d'entendre dans ce phonème son proche homonyme peers, ce qui modifie substantiellement l'analyse. Les pairs désigneraient alors les autres créateurs dont les œuvres embarrassent la mémoire du je poétique, toutes les inscriptions antérieures dont il tente de se délester. Associés à l'obscurité de la nuit, ces pères lunaires incarneraient l'autorité exercée sur le fils, filiation que confirment dans le premier vers les homonymes sun et son (soleil et fils).

Le tissu serré de phrases obscures que brodent les vers, figure donc la mémoire comme l'écran noir qui bloque le passage de la lumière solaire. Émaillée d'images complexes, cette strophe produit un effet de surcharge à la fois visuelle, sémantique et syntaxique. L'accumulation des propositions (tissues the buckwheat, aprons rocks, congregates pears, wakens alleys) aggravée par le poids des syntagmes prépositionnels (in moonlit bushels, with a hidden cough) donne au texte une pesanteur qui s'oppose strictement au dégagement impulsé dans le deuxième hémistiche du deuxième vers (I left in a ravine). Préparé dans le rythme ascendant du premier mètre $I$ left, le départ décrit prend appui sur les deux accents faibles de la préposition et de l'article indéfini qui accélèrent la cadence, et l'envolée s'exprime définitivement dans l'ascension rythmique du iambe final. Ce mouvement prolongé par le tiret est renforcé phonologiquement par les liquides $/ r /$ et $/ v$ / et les fricatives $/ f /$ et $/ v /$ qui suggèrent un passage de l'air, ailleurs bloqué par les allitérations et les consonances des sonorités sourdes $/ \mathrm{k} /, \mathrm{t} / \mathrm{,} / \mathrm{p} /: \underline{\text { Casual, buckwheat, ro }}$

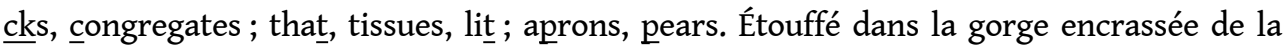
mémoire, la libération du souffle poétique prend dans le dernier vers la forme d'une toux, d'une expiration violente qui dégage des passages (alleys) et correspond au temps de l'éveil.

Dangerously the summer burned

(I had joined the entrainments of the wind).

The shadows of boulders lengthened my back:

In the bronze gongs of my cheeks

The rain dried without odour.

8 La troisième strophe constitue le seuil du poème, la frontière entre le ravin et l'horizon des vallées qui se détache aux vers suivants. Alors que la strophe précédente déployait un espace plein, minéral (rocks), végétal (buckwheat, pears), animal (louse), et meublé d'objets (bushels), l'espace ici décrit est plutôt un anti-lieu, un vide emblématisé par la préposition 
privative without. Des rochers du ravin il ne reste que les formes érodées (boulders) ou plus exactement que les ombres (shadows of boulders), comme si, dans un passage à vide, le monde des substances avait été aboli pour devenir un creuset de l'immatériel : énergie du feu, déplacement de l'air, mouvement vibratoire du son, émanation volatile de l'odeur. Les métamorphoses que subit la matière sont plurielles : réduction des solides par le feu (burned), vaporisation des liquides (le substantif entrainments désigne en effet l'opération chimique qui transforme tout corps liquide en vapeur), assèchement pur et simple de l'eau de pluie (dried). Ce processus atteint également le corps vivant du je poétique dont le dos se déforme (lengthened) et les joues se solidifient en instruments métalliques; métamorphose organique qui efface aussi la frontière des sensations olfactives (without odour), auditives (gongs), tactiles (dried) et visuelles.

Cette instabilité des formes s'exprime encore par le changement perpétuel des sujets (the summer, I, the shadows, the rain) qui sont comme balayés ou détruits les uns après les autres par le souffle puissant ou par la flamme dévorante des vers. Cette substitution permanente s'oppose strictement à la permanence de louse, sujet dans la strophe précédente de tous les verbes, de toutes les actions de liaison (tissues) et de rassemblement (congregates), renforcées syntaxiquement par les enjambements des vers et la conjonction de coordination and. Pour suggérer l'action dévastatrice du feu et du vent, le texte progresse ici davantage par anacoluthe: juxtaposition paratactique de phrases principales, digression et rupture temporelle dans la parenthèse, arrêt du point et des deux points. Enfin, la violence des éléments est amplifiée musicalement par les plosives : allitération de la consonne /b/ dans burned, boulders, back, bronze; répétition de la consonne / $d /$ dans dangerously, dried, odour, effet onomatopéique du nom gongs.

Délocalisante et disloquée, cette strophe est donc à la fois un seuil qui fait passer les choses dans le néant, et une fulgurance. N'ayant "d'aire ni de gîte " comme les «très grands vents » de Saint-John Perse, ces vers ne sont dans le poème que pur mouvement, souffle puissant qui balaye tout sur son passage, démembre le remembrement précédemment opéré par la mémoire. Structure vide, moment de l'oubli, le texte mime le délestage et l'évanescence du sujet qui n'est plus ancré et stabilisé dans le site archéologique et architecturé de sa mémoire.

"It is not long, it is not long;

See where the red and black

Vine-stanchioned valleys-": but the wind

Died speaking through the ages that you know

And hug, chimney-sooted heart of man!

So was I turned about and back, much as your smoke

Compiles a too well known biography.

La strophe suivante arrête néanmoins brusquement ce mouvement, inversant le passage en impasse. L'espace indifférencié des vers précédents se restructure dans les contours d'une vallée et autour d'axes verticaux que dessinent les piquets (stanchions) soutenant les branches des vignes et le conduit de la cheminée (chimney). Les vers auparavant déliés sont réarticulés par les conjonctions but, and et so, par les prépositions through, that et as, et par les enjambements. Ce retour des lignes spatiales et des figures de liaison reflète la reconstitution du poète confronté à l'impossibilité de sa propre déstructuration. Ainsi peut-on entendre dans l'homonyme heart le prénom Hart (Crane signait souvent ses lettres en dessinant un cœur) qui désigne le poète en tant qu'homme ancré dans l'Histoire et dans son histoire. Pourtant, cette personne ne coïncide plus avec le sujet poétique I, 
mais avec le pronom you (the ages that you know, your smoke compiles a too well known biography) dans une scission identitaire que vont développer les deux strophes suivantes.

The evening was a spear in the ravine

That throve through very oak. And had I walked

The dozen particular decimals of time?

Touching an opening laurel, I found

A thief beneath, my stolen book in hand.

"Why are you back here-smiling an iron coffin?

"To argue with the laurel," I replied:

"Am justified in transcience, fleeing

Under the constant wonder of your eyes"

Dans la cinquième strophe, la forme interrogative de la phrase And had I walked the dozen particular decimals of time? introduit une ironie dans l'énonciation, une duplicité qui met en doute le retour du je poétique dans le ravin de la mémoire, sa retombée dans le temps et sa biographie. Le dialogue entre le je et le voleur accentue davantage encore cette division discursive : il n'y a plus, comme au début du poème, une unité du sujet, mais deux êtres. L'un est garant du livre biographique (my book), de la mémoire, des inscriptions antérieures; l'autre cherche les lauriers de la gloire poétique (laurel) et son salut dans le fugitif et l'éphémère (transcience) et contemple ironiquement le passé recueilli dans un tombeau (smiling an iron coffin). L'image guerrière de la lance perçant le chêne, et l'ellipse de l'article dans le syntagme very oak confirment que l'arbre, figure de la généalogie, n'est plus aussi puissant et intact que dans la deuxième strophe puisqu'il est à présent mutilé et fendu. L'espace du ravin n'est donc plus celui qui ouvrait le poème, et cette différence est encore signalée par la métrique, par la présence du pentamètre associé précédemment à la promesse de silence antérieur au langage (I was promised an improved infancy), à la première envolée (My memory I left in a ravine) et au vent subversif de l'inspiration poétique (I had joined the entrainments of the wind). Terrain d'une friction entre le moi et l'autre, l'ouvert (opening) et le fermé (coffin), le continu (constant) et le passager (fleeing), lieu d'un affrontement que résume le verbe to argue, ces vers préparent le dénouement final de la dernière strophe du poème.

He closed the book. And from the Ptolemies

Sand troughed us in a glittering abyss.

A serpent swam a vertex to the sun

-On unpaced beaches leaned its tongue and drummed.

What fountains did I hear? what icy speeches?

Memory, committed to the page, had broke.

13 La clôture du livre, l'éclatement de la page et de la mémoire réalisent enfin la promesse de la parole originelle énoncée en ouverture. Équivalent phonique de cette parole dans la première strophe (sea, sapphire, promised, infancy), la sifflante envahit avec une intensité accrue l'espace sonore : sand, us, abyss, serpent, swam, sun, unpaçed. Phonème identique au sifflement du serpent, cette consonne est doublement associée, par sa graphie serpentine, au reptile qui, dans ses mues successives, figure le délestage perpétuel du passé ou l'éternel recommencement. Cette image est en tous points semblable à celle qu'Emerson convoque dans le premier chapitre de son essai sur la nature : «the lover of nature [...] has retained the spirit of infancy [...] In the woods too, a man casts off his year, as the snake his slough, and at what period soever of life, is always a child. In the woods is perpetual youth $»^{4}$. À cette jeunesse permanente de l'homme, Crane ajoute cependant l'idée d'un renouveau continuel du langage que permettent, dans cette strophe, les différents néologismes. Réfutant leur sens archaïque établi et autorisé, le poète promet 
aux mots une nouvelle naissance, accorde à trough de nature purement nominale la qualité inédite d'un verbe, transforme le verbe swim qui devrait être ici intransitif en verbe transitif, crée un participe original en ajoutant à paced déjà existant le préfixe privatif un. Les plages vierges (unpaced beaches) et les paroles gelées (icy speeches) peignent ainsi, en creux, l'évidement du langage qui précède sa régénération dans le verbe musical, tambourinant (drummed) ou cristallin (what fountains did I hear?) de la poésie. Ce passage du vide au plein est assuré dans la structure chiasmique de ces vers et plus haut dans l'abîme lumineux (glittering abyss), figure imposée et librement conjuguée dans une série de variations oxymoroniques : fluidité des fontaines et solidité de la glace, hauteur (vertex, sun) et creux (troughed, abyss), eau (swam, fountains) et feu (sun), union (committed) et éclatement (broke), affirmation (leaned its tongue and drummed) et interrogation (What did I hear?).

14 Ce poème accomplit ainsi le rêve de Crane qui était de donner à son lecteur « un mot nouveau, jamais dit auparavant et impossible à énoncer », un verbe toujours inédit, fugitif et passager, une parole "protéenne" qui contre l'inertie du sens, arrêté par la grammaire. Il constitue aussi un prélude aux échappées plus ambitieuses que réalise la longue séquence textuelle, placée en clôture de « White Buildings » : « Voyages ».

\section{NOTES}

1. Voyager: A Life of Hart Crane. New York: Farrar, 1969.

2. "Passages, Margins and Poverty: Religious Symbols of Communitas", Worship 46 (Aug.Sept. 1972), 401.

3. «Let us invent an idiom [that's] elusive.» The Letters of Hart Crane (1916-1932), New York: Hermitage House inc., 1952, 89.

4. Ralph Waldo Emerson, « Nature », Complete Works 2. London: Bell and Dalby, 1866, 142.

\section{INDEX}

oeuvrecitee Passage 